\title{
Near Chaos in Graphic Drawings from Analogue Machines
}

\author{
Jack Tait \\ 1 Green End, Hereford Road \\ Mardy, Abergavenny, NP76HT, Wales \\ jack.tait@btconnect.com
}

\begin{abstract}
Chaos is now recognised as one of three emergent topics of study in the 21c. It is seen as appropriate to examine this in art practice. The paper examines the author's recent research into 'near chaos' and describes how the original default position i.e. 'simple instructions leading to complex images' has been extended. The intent is now to disrupt the programming input, moving the drawings towards 'near chaos'. The tipping point is approached where an image almost descends into chaos but just remains visually coherent. Evaluation and philosophy are discussed. The Power Point presentation will include practical and theoretical underpinning, and detail the workings of the machines, via still and video images.
\end{abstract}

Chaos. Determinism. Quasi-randomness. Analogue drawing machines. Machine art. Graphic characteristics of broken lines

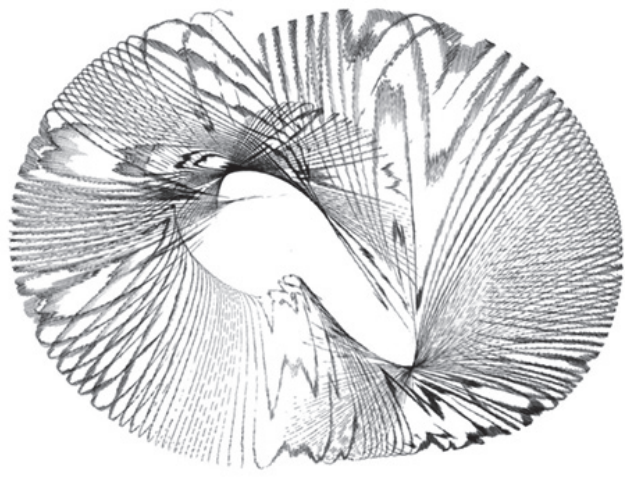

Figure 1: HHM drawing where determinism is moving to 'near chaos' in the form of secondary patterns.

\section{INTRODUCTION}

As Chaos has emerged as a topic of study in the 21c (Gleick 1987), the paper attempts to extend it to art practice. The basis for the research centres on a recent $\mathrm{Ph}$. $\mathrm{D}$, 'Programmable Analogue Drawing Machines' awarded by Manchester MIRIAD in 2011 (Tait 2011). This led to 'Art by Machine' (Tait 2013) summarizing the research, and work covered in the author's website (Tait 2017).

Past work with analogue drawing machines has concentrated on simple instructions leading to complex outcomes and inspired by Turing's work in the mid 20c. (Turing 1952). The intent was to produce non-figurative art works, concerned with striking a balance between determinism and quasirandomness.

The approach is now modified; further complexity added to enable study of 'near chaos'. Increasing complexity might be expected to make it less likely to achieve coherence in the images. However, even allowing for the subjective nature of art evaluation, the outcomes have been better than expected and are discussed in section 3 . Coherent images persist and curiosity about it is the motivation.

Designing drawing machines, to investigate 'near chaos', is counter intuitive. Less accuracy, causing unpredictability, might seem helpful but the opposite is true. The basic machine has to be deterministic, precise in line-to-line accuracy, and place one $0.5 \mathrm{~mm}$ line 1 to 1.5 line widths from the next with consistency over a long timescale (Figure 2 ). With this, any deviation from a deterministic starting point may become more apparent. A 'near chaos' result, created by extra mechanical devices and programming can then be judged by its distance from a predetermined starting point.

To create 'near chaos', a process of graphic disruption is necessary; combining mechanical 
actions to increase complexity. Before these are listed, the various machine types are described to which the actions are added.

In the basic machine determinism starts with the different mechanics of pen movement and control. The machines to which they are applied may be any of the following.

Two machines, the HHM and NTT draw a simple Lissajous figure, which grows organically (Figures 11 and 12).

In the NSEW ch machine a set of Cartesian coordinates are input to an $X: Y$ plotter which is coupled to rotary pen motion and pen lift (Figure 6).

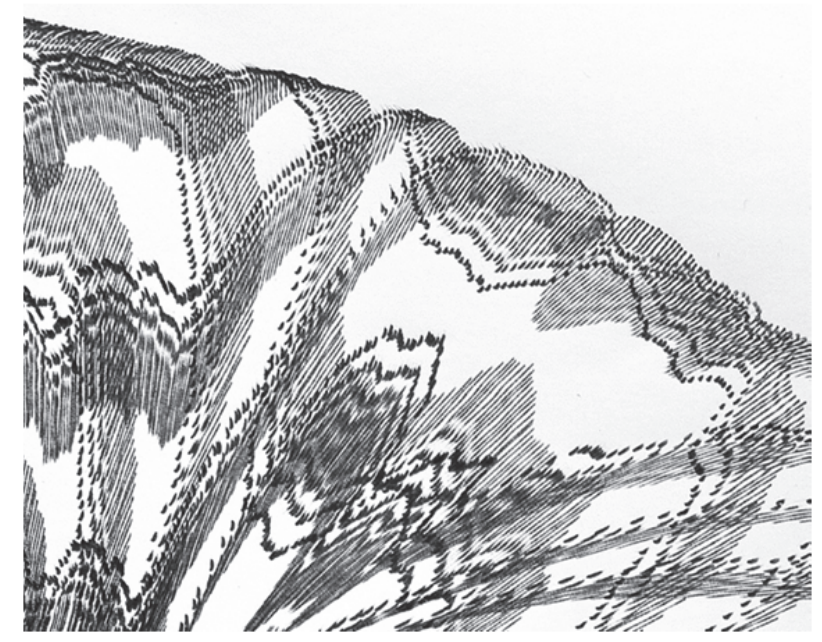

Figure 2: HHM detail from Figure 1 showing line-to-line accuracy.

The Sinewave machine is a rotating drum design drawing sine waves with variable amplitude, as well as using pen lift and modifying the wave form (Figure 16).

The Camera Moving Machine has a static light source recorded on a moving digital camera (Figure 7). Colour and tone are overlaid, subject to two-dimensional movements by sun and planet drives. This machine is very different from the others in output and operation and evaluation.

The disruptive sequence of actions are listed below. Breaking up the continuous line into dots and dashes causes secondary patterns, implying lines at angles to the primary ones (Figure 2). D.C motors driving the machines 'hunt' under load and temperature causing variations in speed, which creates a quasi-random input.

Sun and planet gearing systems are interposed between the motor and linkages and together generate complex mathematical curves (Figure 5). The type of linkage used influences the drawing with its non-linear geometry effecting the way the pen reacts to the programme.

When separate D.C motors drive the axes via sun and planet gearing driving, and also control the pen lift and rotation there can be six motors working at varying speeds under different load conditions. The sum of these elements alone is sufficient to create a lot of quasi-randomness. A recent modification added to the NTT and NSEW ch machines are two pen units to further extend the potential. Finally, where a time sequence programmer is employed, using a seventh D.C motor, the combination increases the potential for a chaotic outcome.

Other factors may increase complexity. An NSEW drawing is usually scanned and colour added in Adobe Photoshop (Figure 23). The addition either reinforces a deterministic outcome or alternatively pinpoints the near chaotic aspects of the drawing (Figure 17).

Overall graphic chaos in art is seen in relation to its opposite, order. The debate involves intent, design and philosophy. The next section shows the machine workings and highlights the elements contributing to chaotic drawing. The final section concludes with a detailed discussion of images from each machine addressing the philosophy of 'near chaotic' images in art.

\section{THE MACHINES}

Writing this paper meant re-examining past work to find those with 'near chaos' characteristics and also meant redesigning machines to increase their potential to exhibit chaos. Many machines have been built during the author's research and a small number of current ones have been chosen.

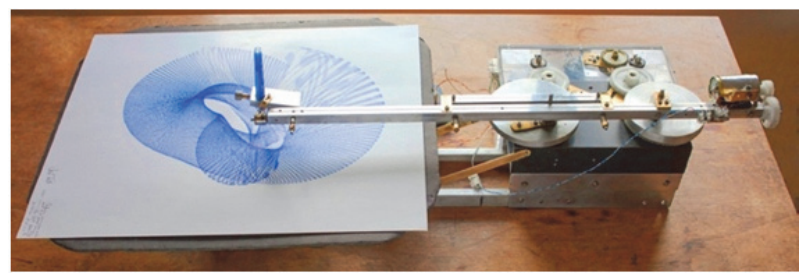

Figure 3: HHM machine, with an elliptical platen and sun and planet drives for the $X$ and $Y$ axes. $X: Y$ speed difference is governed by a differential gearbox; $X: Y$ ratio 1:1.002. See Figure 1 for image.

\subsection{HHM machine}

Five current machines are described. The HHM (Hommage to Henry Machine) was reverse engineered to recreate Desmond Paul Henry images from the 70s at Manchester based on his daughter's research (O'Hanrahan 2005). It is capable of high of line-to-line accuracy (Figure 2) creating a deterministic starting point where the 
slightest move towards chaos is easily seen. Another image (Figure 10) below shows dot patterns and longer dashes and led to the later machines increasing their accuracy and range as in (Figure 11).

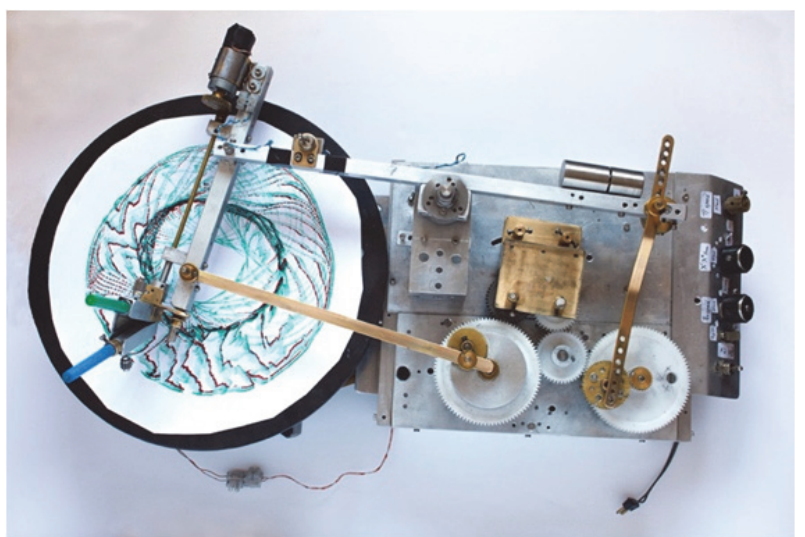

Figure 4: NTT machine specially developed for 'near chaos'study. The suns and planets are coupled, the pair driven by two matched D.C. motors, which slowly get out of phase. Relative motor speeds, pen lift and turntable are programmable. The latter is a separate unit and an alternative linear platen is under design.

\subsection{The NTT machine}

The NTT machine (New Turn Table machine) was designed to explore graphic chaos for a special issue of the MDPI mathematics magazine (Tait Jack 2019). The NTT and the HHM machine are Lissajous figure generators where the organic growth of the drawing has very close line to line conformity showing progression from determinism to near chaos. Both have integral programming with $X$ and $Y$ axes driven by sun and planet gearing. These produce complex curves particularly when separate motors drive each part and contribute to creating 'near chaos'. In addition to the sun and planet drives the NTT has different linkages, which also influence the graphic character.

The NTT machine differs from the HHM in that it can employ two colour pens, which can be of different thickness, and draw lines in close proximity. This creates another move in the direction of a chaotic outcome. The engineering problem is to ensure each pen stays in accurate contact with the paper and when both are lifted and lowered the contact is synchronous. This is a very recent development.

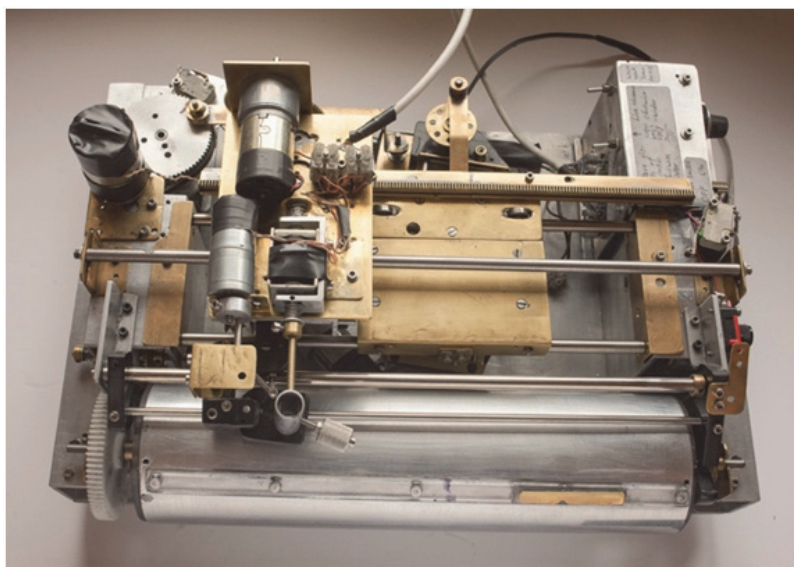

Figure 5: Sinewave machine with organic waveform growth, pen lift and 'waveform modifier'.

\subsection{Sine wave machine}

The third machine draws sine waves with organic growth and decay of wave amplitude (Figure 14). Line to line proximity and accuracy is essential. Overlaid over waveforms, a pen lift creates broken lines working alongside an optional 'wave form modifier'.

The modifier device is designed to distort the waveform at points along its progress in order to mimic a wave break effect. Both push the drawing into a near chaotic state. The Sinewave machine has integral programming.

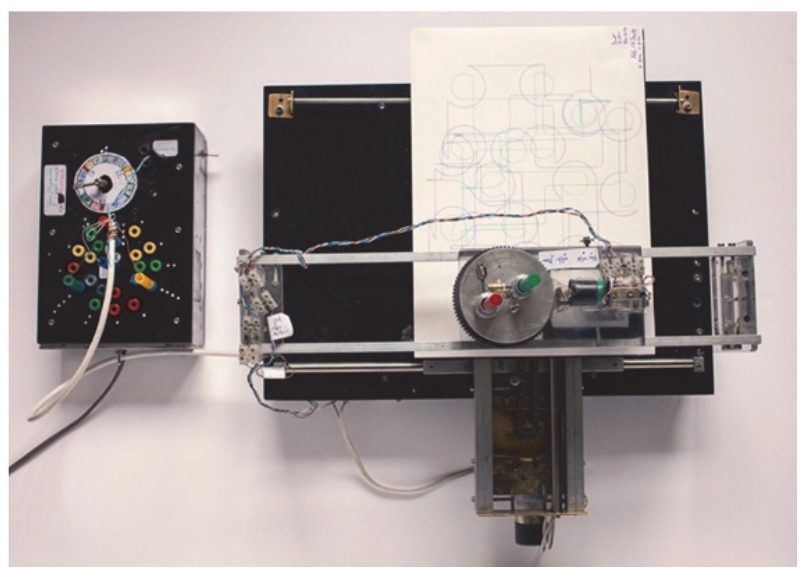

Figure 6: NSEW ch is an X:Y plotter with programmer. In the centre a pen rotator and pen lift can be seen. 


\subsection{NSEW ch machine}

This is an $X: Y$ plotter (now in a Mk IX version) where the pens rotate and lift. NSEW stands for North, South, East, West i.e. the directions available and 'ch' indicates chain driven axes. It is governed by an analogue programmer, sending out timed pulses to the axes, pen lift and pen rotation. The pen can draw in two colours, the pen lift allows a broken line and is able to rotate. Enhancement in Adobe Photoshop, with extra colours added to closed areas differentiates between lines and area, which identifies progress towards 'near chaos' (Figure 17).

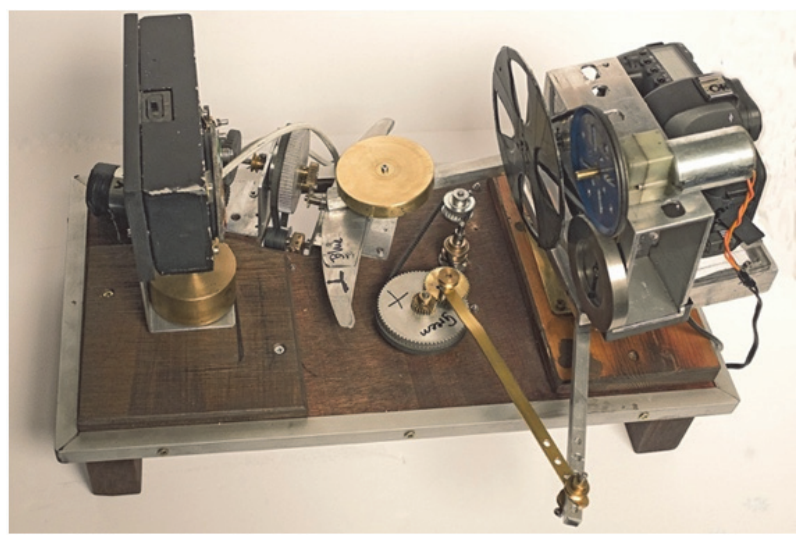

Figure 7: Swinging counter balanced camera platform with high-speed shutter unit with one of the slit light pens on the left.

\subsection{Camera Moving machine}

The fifth machine is very different from the graphic ones. The $X: Y$ movements of a digital camera record a stationary light pen. The camera platform has a high speed shutter switched on when needed. Three light pens are available; one has a spot image (Figure 8), which changes size and colour as the drawing progresses whilst two others rotate different size slits. The sections along the large slit have fixed colour gels; in the small slit the colours vary as the slit revolves.

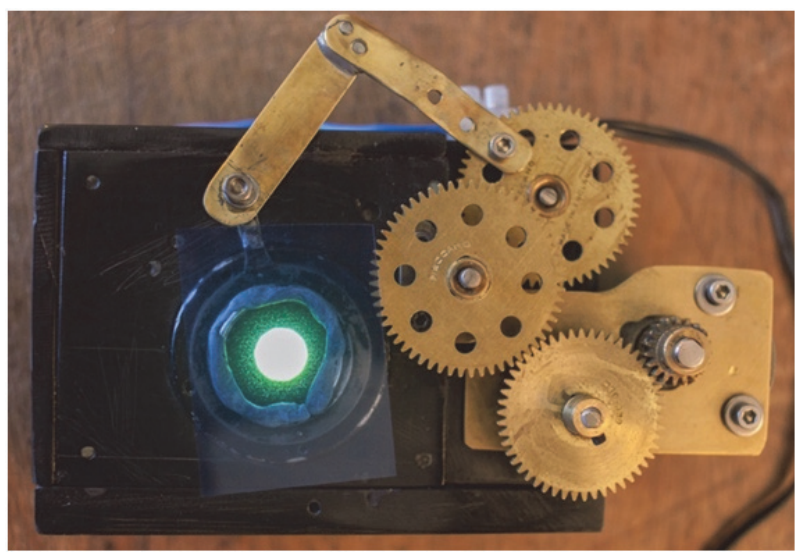

Figure 8: Light pen variable spot and automatic colour change.
Using and programming the Camera Moving machine is more complicated and slower than the graphic machines and of course the drawing is done in the dark.

'Near chaos' light images have a different character. The aesthetic qualities are based not on secondary patterns and line-to-line effects but on overlaying colour and tonal differences. As stated above this alters the evaluation as the image is seen more as a photograph than a drawing. The effect this has on perception is touched on in Section 3.

The two axes in the camera moving machine are driven by sun and planet cranks (Figure 4). Each of the four actions has its own motor where individual control increases the potential complexity.

The overall control of the camera movement is via the same programmer as the $X: Y$ plotter (Figure 9).

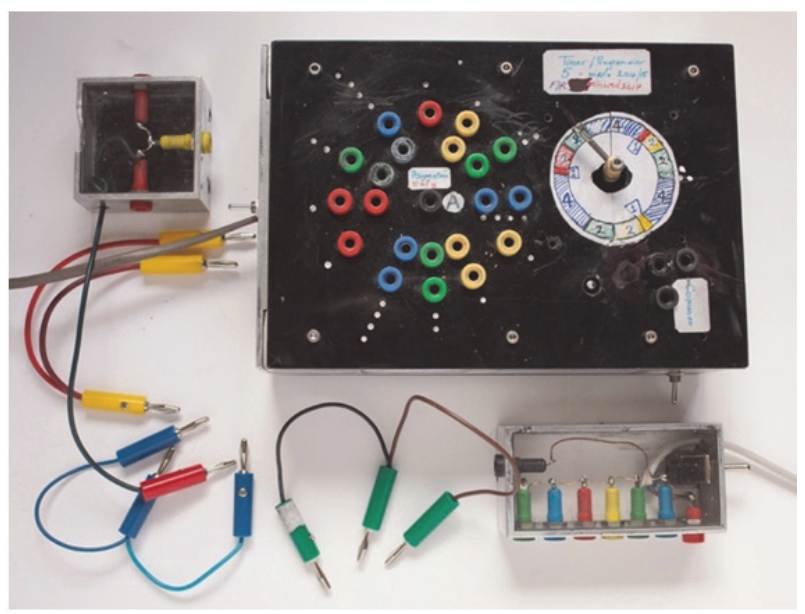

Figure 9: The programmer with distribution boxes and extra leads.

The sequential time programmer is fundamental to the NSEW and Camera movement machines. The HHM, NTT and Sine wave machines have integral programming. The programmer outputs varying time sequences in recurring sets 1,2 or 4 in a fixed order and the different axes, pen lift/rotate actions are under its control.

Flying leads allow a very large number of permutations of instructions. The distribution boxes are needed when a number of units have to be hooked up. In the middle of the main box is an asymmetric outlet delivering pulsed times independently of the main rotational sequencer.

The extra asymmetric outlet significantly increases the number of programmes available and also changes the character of the drawing. Two different actions will get out of phase, which would not otherwise be possible; the main motor alone has fixed sequences. 


\section{EVALUATION, ANALYSIS AND PHILOSOPHY}

The approach to the art works follows the Gombrich (1960) notion of the 'beholder's share' i.e. the response to and benefit derived from an artwork is governed by what the viewer brings to the process. Whilst this puts the artist's intent into second place it is felt to be a workable practice and is adopted below.

Evaluation is more complex when the subject is 'near chaos'. As mentioned in section 1 above it is the opposite end of a continuum between itself and order: the theme of Kenneth Martin's 'Chance and Order' work in the mid 20c. (Martin 1975). His work explored the role of chance. Using dice as random input, images displayed 'near chaos' characteristics.

The continuum establishes a deterministic start showing the distance moved towards 'near chaos'; the process ending when coherence is evident. Evaluation is informed by the expectation that combined inputs should fail to generate coherent images. Surprisingly this has not been the case and near chaotic images are discussed below.

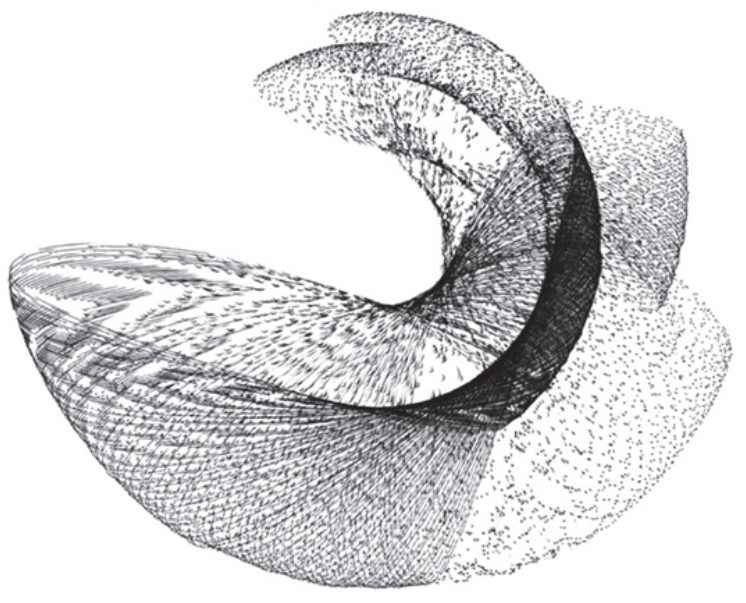

Figure 10: An early HHM drawing indicating 'near chaos' effects, which encouraged further research. The pen lift and the D.C motor's instability created the drawing's changing character.

The HHM machine drawings, by virtue of their line accuracy, illustrate the point made above regarding moving along the continuum towards chaos but stopping at a coherent point. The original is large, A2 size, and the detail may not be appreciated at this scale; an idea of this is in (Figure 2). A recent modification has been to put a sun and planet drive on both axes to increase the richness available. The main feature of the $X ; Y$ ratio, which produces the accuracy, is the differential gear box which may be set as close as is required. This sets a high initial determinism level allowing very small deviations to be seen. (The NTT $X: Y$ ratio relies on the 'hunting' properties of two D.C motors', which offer an alternative albeit subtle variation.) This programming creates welcome but unexpected secondary patterns and set the scene for further research into 'near chaos'.

The NTT machine was built to explore the graphic effects of different linkages (to the HHM) coupled with the complex curves of sun and planet drives. The mechanical detail has to be addressed in order to relate it to the original concept.

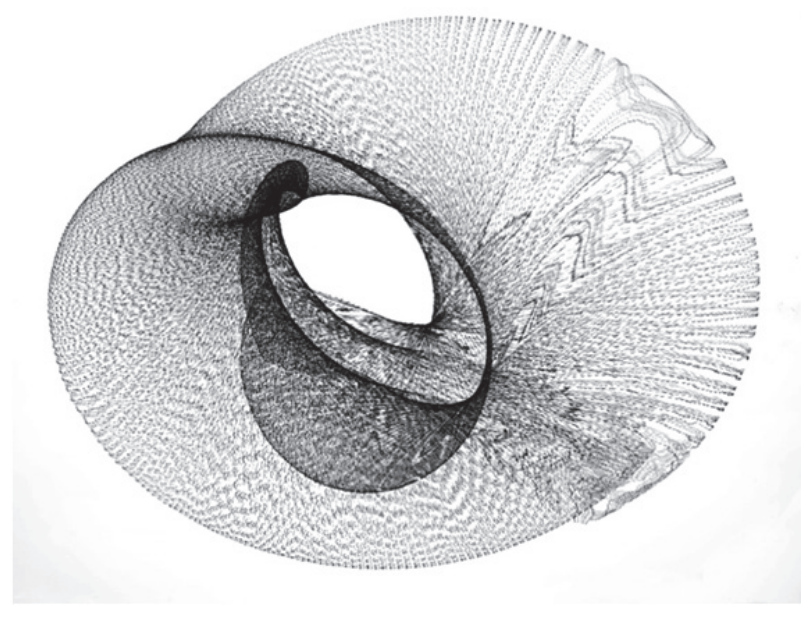

Figure 11: This HHM image shows a variety of secondary patterns and an indication of "near chaos, qualities.

The NTT with sun and planet $X: Y$ axes has an ancillary gearbox, where the relative directions can be permutated, to give the greatest range of options. The relative direction of the axes exerts a significant influence on the drawings.

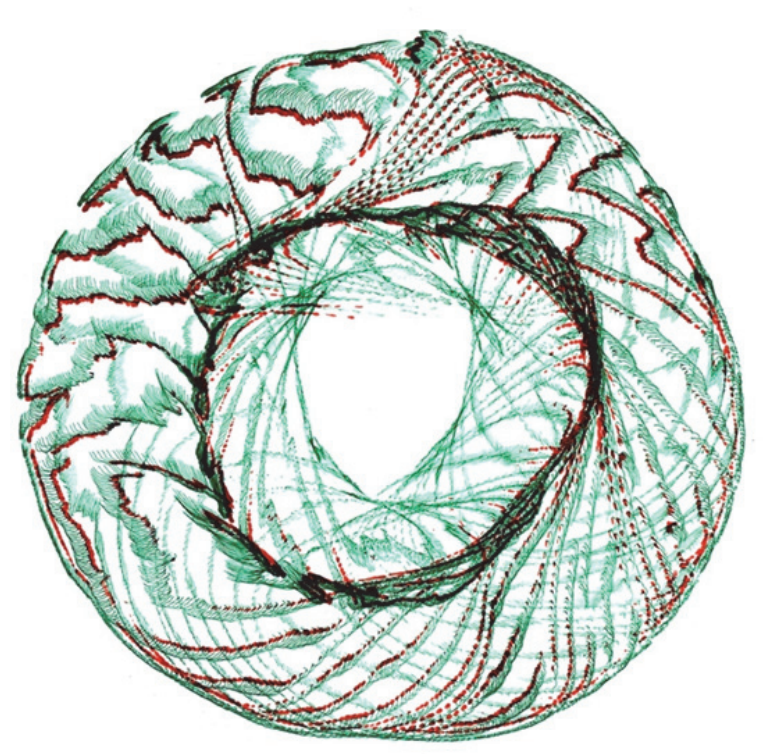

Figure 12: NTT two pen image with differing pen thicknesses. 


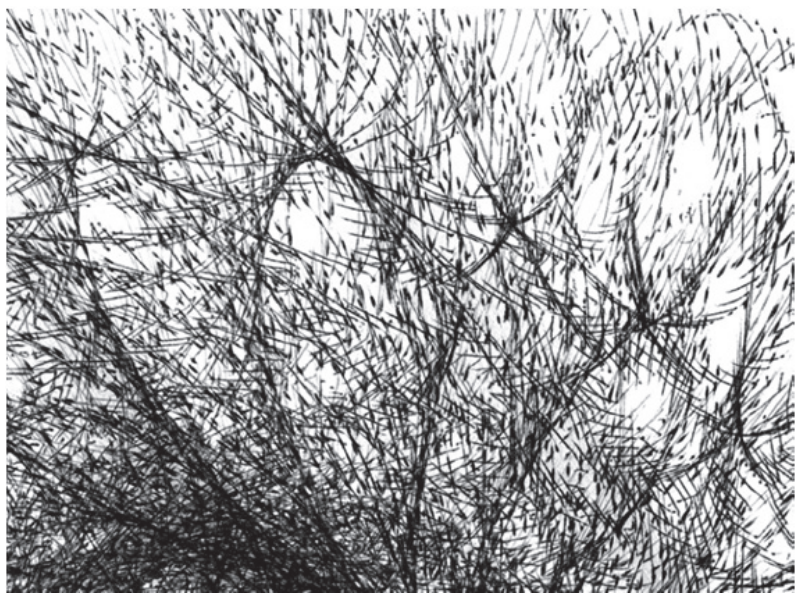

Figure 13: A section of the drawing is close to the limit of coherence

The linkages are finely counterbalanced to reduce friction. This allows any chaotic elements to emerge, which might be caused by motor speed differences as well as pen and linkage geometry. Whilst quasi-randomness is an important aspect of the design it must be channelled to follow the initial idea termed by the author as 'the fuzzy notion of intent' (Tait 2011). The next aspect is the pen lift action; its rate of lift in relation to the axis speeds is important and it must not inhibit the linkage balance. Finally two pens, in close proximity, make a significant contribution, adding a feature at present missing from other machines.

Regarding presentation of the final image and its evaluation, Figure 13 shows that an enlarged detail of a drawing may be a good choice as it shows 'near chaos' effects to advantage.

This drawing is on the cusp; parts are chaotic and others just coherent. The NTT machine went through a few redesigns but finally succeeded in exploring graphic chaos. The concept was clear but the achievement difficult. As with the HHM machine, precision and accuracy were crucial.

The Sinewave machine's notion, as stated above, is based on sinewaves and builds in varying amplitude. Sinusoidal shapes are important mathematical figures, and are very different from Lissajous figures. This deterministic base allows variations from the expected geometry to emerge. A separately driven pen lift, added to a wave form modifying device, facilitates 'near chaos' exploration.

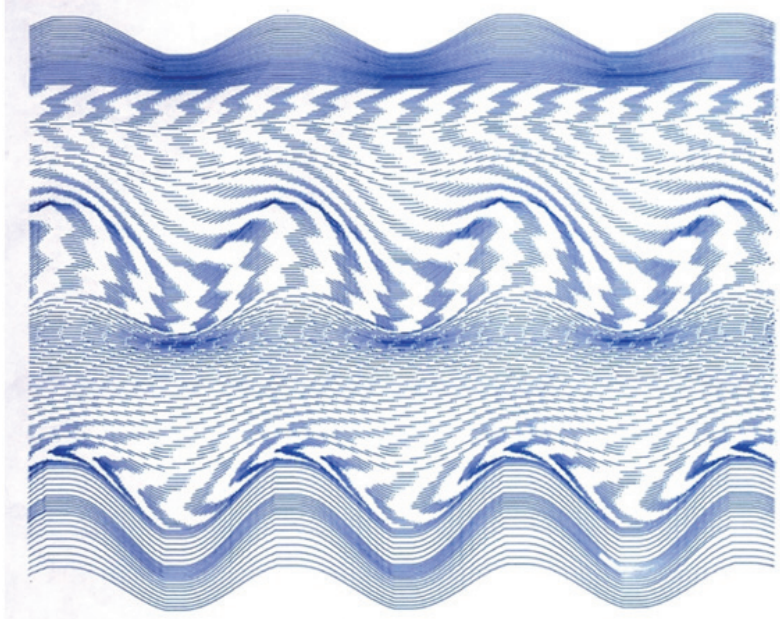

Figure 14: Sinewave image with both solid and broken lines but no wave form modifying device operating. It allows interactive control whilst the drawing takes place. This picture shows the variety of effects available.

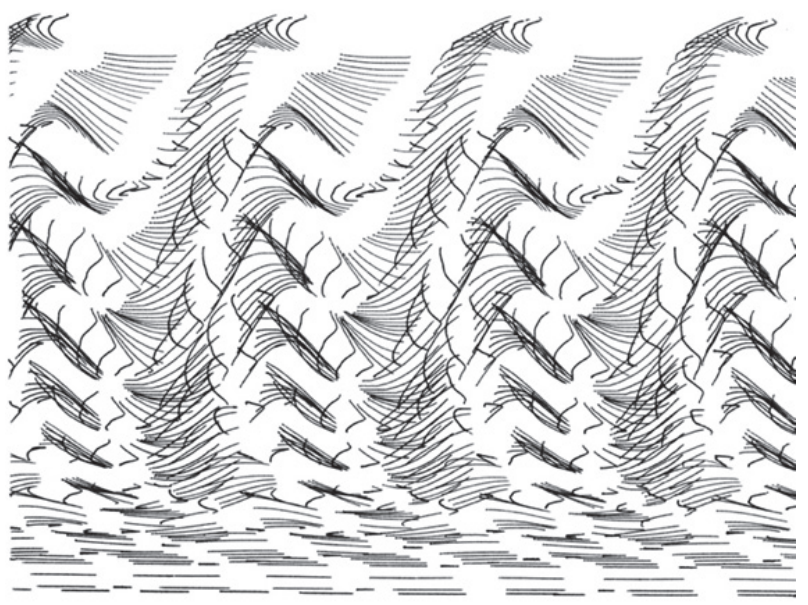

Figure 15: Illustrates the deviation from the basic sign wave in the direction of a more chaotic outcome.

Although the sinewave is a simple shape it allows a large variation in drawings to be achieved. The frequency variation in the pen lift speed, relative to the sinewave generator, alters the length of the line from a dot to a long stroke, whilst an 'out of phase' wave form modifier helps to extends the range further.

The NSEW ch machine: a basic $X: Y$ plotter, now seen mainly in digital form, is the basis for many versions of drawing machines and is usually controlled by a programmer. 


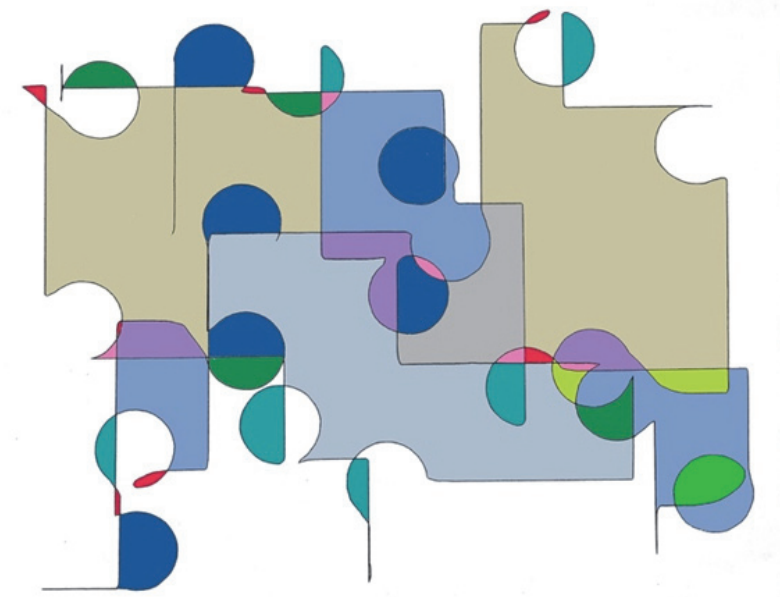

Figure 16: Showing circular additions to the $X: Y$ directions. Colour is added to highlight the circular and linear blocks. Near chaotic quasi-random distribution of the circles is evident.

The NSEW ch machine is enhanced by a rotating 'two pen' holder, where each pen draws a different diameter circle. The pens can also be lifted so that the pen unit can add curves to the straight lines of the $\mathrm{X}: Y$ directions.

This rudimentary combination produces many variations, generating complexity from simple instructions. It differs from other machines, allowing another approach to 'near chaos'. In this case the drives are linear, hence the 'ch' suffix denoting a chain drive. Cranks differ being non-linear.

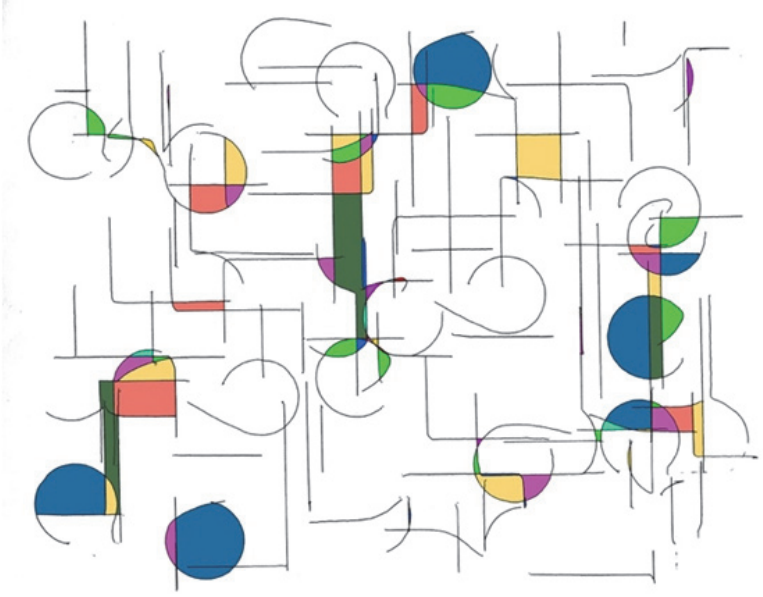

Figure 17. The circle plus $X: Y$ lines with pen lift allows 'near chaotic images to be made.

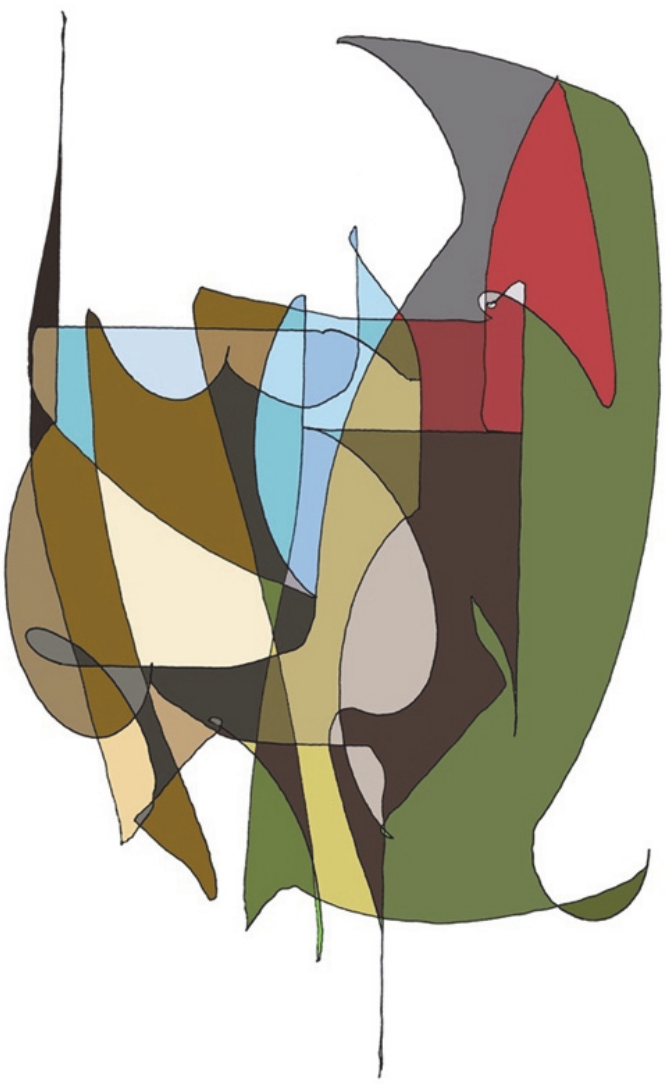

Figure 18. Other versions of the NSEW machine have employed non-linear sun and planet drives to take advantage of the different geometry of complex curves. Colour added in Adobe Photoshop.

The Camera Moving machine draws with light, using different light pens, with circular and slit shaped sources. The colours are either fixed to a large slit source or change automatically. In one case the spot images changes size as the drawing progresses.

The camera's movement is driven by sun and planet systems, and an ancillary high-speed shutter is mounted over the lens. Control is by the analogue programmer with digital results treated as photographs in Adobe Photoshop. 


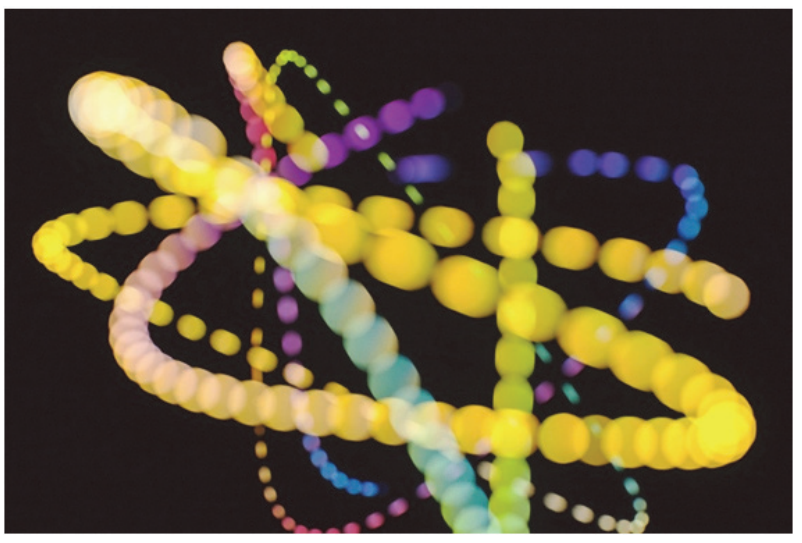

Figure 19. Spot image changing both colour and size. A shutter was switched on to separate the dots.

With light images, tone and colour replace graphic pens, whilst the 'line' is broken by using a shutter. Previously any manifestation of 'near chaos' was judged as a distance from a deterministic starting point. With light traces on a black ground. It is not easy to establish a starting point.

What remains is to evaluate the coherence or lack of it from an imaginary standpoint. One image may exhibit a different level of coherence from another.

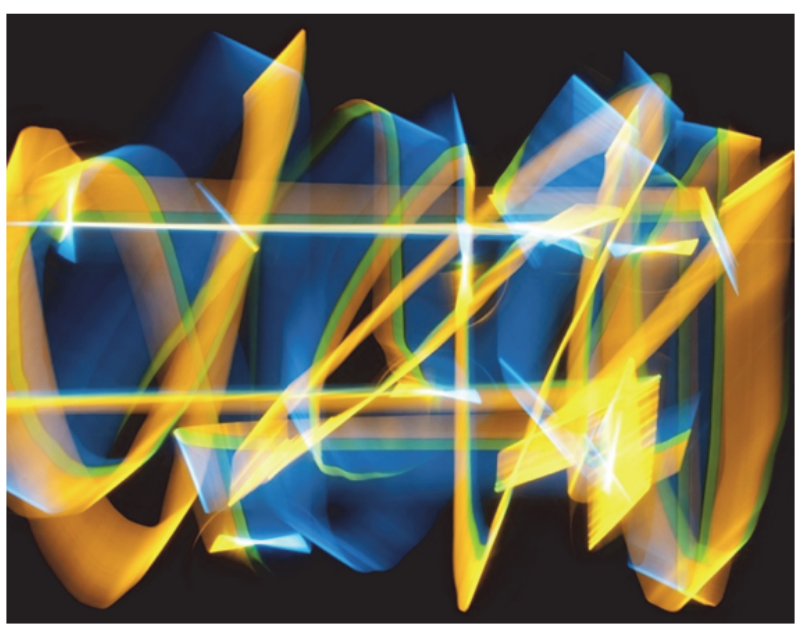

Figure 20: Slit image with similar colour range to Figure 19 It could be judged as more chaotic.

To further emphasise this point, two more images are shown, where differences in the amount of 'near chaos' may be discerned. The first is effective in using subtle tonal gradations and colour contrasts to create what could be seen as an aesthetically pleasing image. If this is the case it may well militate against the evaluation of an image, which is meant to explore 'near chaos'.

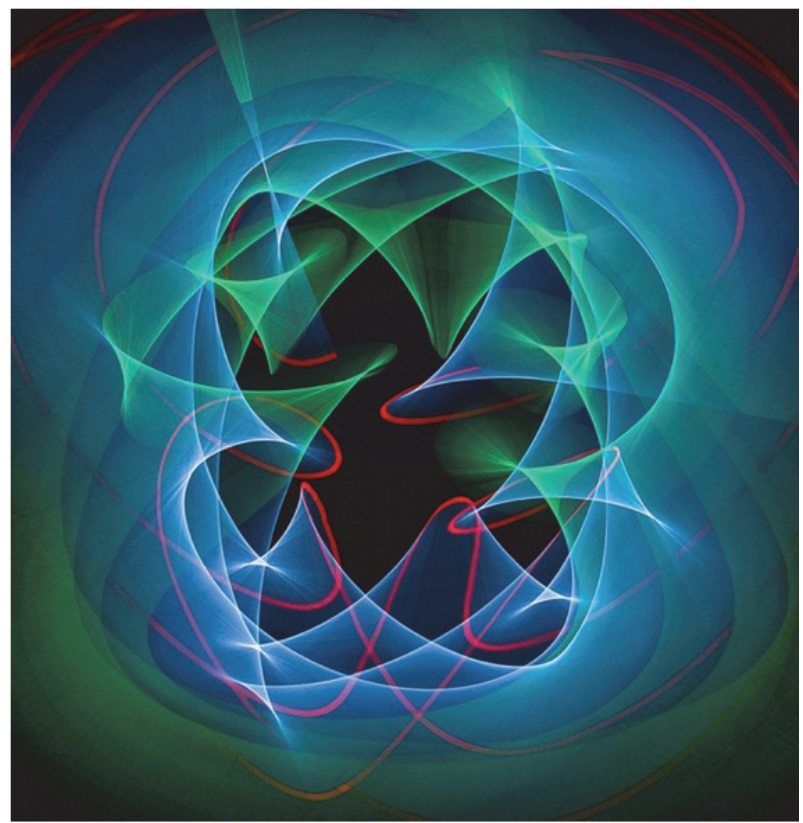

Figure 21: A well-structured image expected to be chaotic has the appearance of coherence and order.

The above image is contrasted with the one below. Both formed using a wide slit light source with fixed colour gels. The second differed in that a highspeed shutter broke up the broad line made by the large slit source.

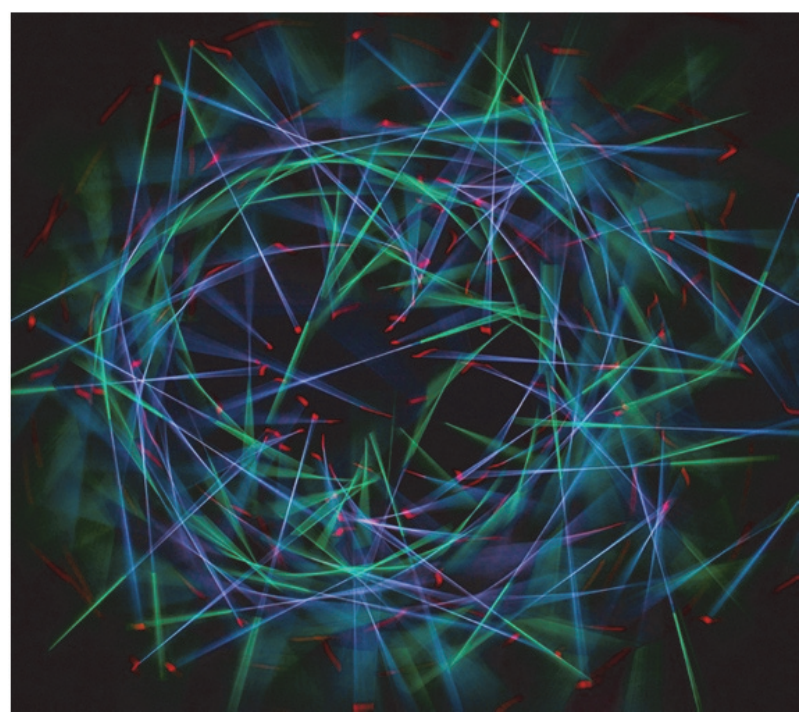

Figure 22: Given similar beginnings, the image is a step nearer chaos. A high-speed shutter was used which changed the result.

Figures 19-22 also imply a possible conflict, bearing on the process from intent to final selection. Figures 19 and 21 are either less far along the continuum postulated above or alternatively they diverge at some point and change character? It is difficult to define which is most relevant. 
Evaluation of chaos and art intensifies the problems of definition, addressing two complex issues. The complexity calls for a philosophical approach and factors below come into play. Considering them might clarify the issues.

Our predisposition to seek figurative meaning in abstract images occurs in Hamlet and Polonius's argument about the shape of clouds, and has a bearing on our responses today. In Figure 18 a product from quasi-random input has been perceived as a woodpecker. A drawing programmed with chaotic potential has become an illustration!

The view can be taken that the artist's job is not to convince but to offer results stemming from curiosity and seeking innovation. Then re. 'Gombrich', the viewer makes of it what they will. Does this render intent irrelevant? Another viewpoint comes from the philosopher Collingwood, who stated that "Art is the community's medicine for the worst disease of mind, the corruption of consciousness" (Collingwood 1958).

Paul Klee (Klee 1997) was a musician and artist so a musical analogy might be helpful. Music consists of notes of different pitch and timbre, where delivery and interpretation govern the effect on the listener. The same music has first been seen as Chaotic and eventually as Classical. Art objects now seen as chaotic may be seen differently in future although the artist's curiosity remains the same.

A concluding thought is offered. Simple instructions with a potential for chaos created (Figure 23) with complex lines and added colour. The author sees 'Homage to Klee'; others may see a jumble of lines with no meaning.

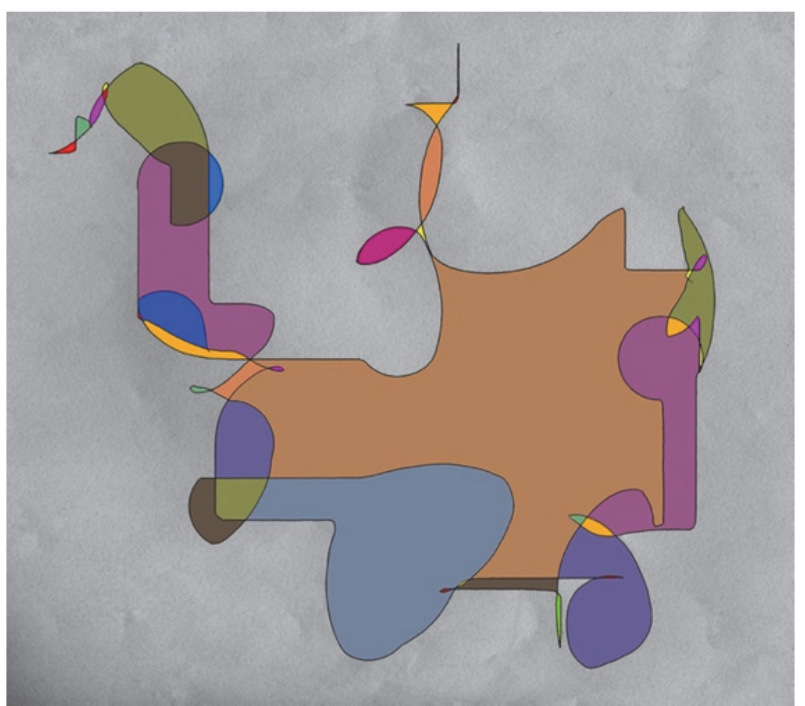

Figure 23. NSEW ch drawing, $X: Y+$ circular motion.

\section{REFERENCES}

Collingwood, R. J. (1958) The Principles of Art. Oxford University Press

Gleick, J. (1987) Chaos, The amazing science of the unpredictable. Vintage, London.

Gombrich, E. H. (1960) Art and Illusion: a study in the psychology of pictorial representation. Phaidon, London.

Klee, P. (1997) Painting Music. Prestel Verlag, Munich.

Martin, K. (1975) Chance and Order, Tate Gallery.

O'Hanrahan, E. (2005) Drawing Machines: the machine produced drawings of Desmond Paul Henry in relation to conceptual and technological developments in machine-generated art (UK 19601968). Unpublished MPhil thesis, Liverpool John Moore's University, UK.

Tait, J. (2011) Programmable Analogue Drawing Machines. PhD thesis MIRIAD, Manchester Metropolitan University.

Tait, J. (2013) Bronydd Press, Clyro.

Tait, J. (2017) http://taitographs.co.uk (retrieved 12 March 2019).

Tait, J. (2019) 'Secondary Near Chaotic Patterns from Analogue Drawing Machines.' Special Issue Topological Modeling Mathematics, 7(1), January 2019.

Turing, A. (1952) The Chemical Basis of Morphogenesis. Philos. Trans. R. Soc. Lond. 237, doi:10.1098/rstb.1952.0012. 